\title{
Development of a Microwave Cusp Accelerator Driven by Electron Cyclotron Resonance
}

\author{
Bradley S. Sommers ${ }^{1}$, John E. Foster ${ }^{2}$ \\ University of Michigan, Ann Arbor, MI, 48104
}

\begin{abstract}
A compact microwave ECR source has been developed to provide gridless acceleration of ions for discharge voltages up to $80 \mathrm{~V}$. The device employs a magnetic cusp created by permanent magnets, which simultaneously provides the resonance condition for ECR absorption and establishes the ion acceleration zone by impeding cathode electron flow to the anode. Ion energy measurements with a three grid energy analyzer, coupled with plasma potential measurements outside of the source, indicate the presence of ion beams with a significant fraction of the discharge voltage. Ion saturation current measurements indicate current densities near $20 \mathrm{~mA} / \mathrm{cm}^{2}$ immediately outside the device.
\end{abstract}

\section{Nomenclature}

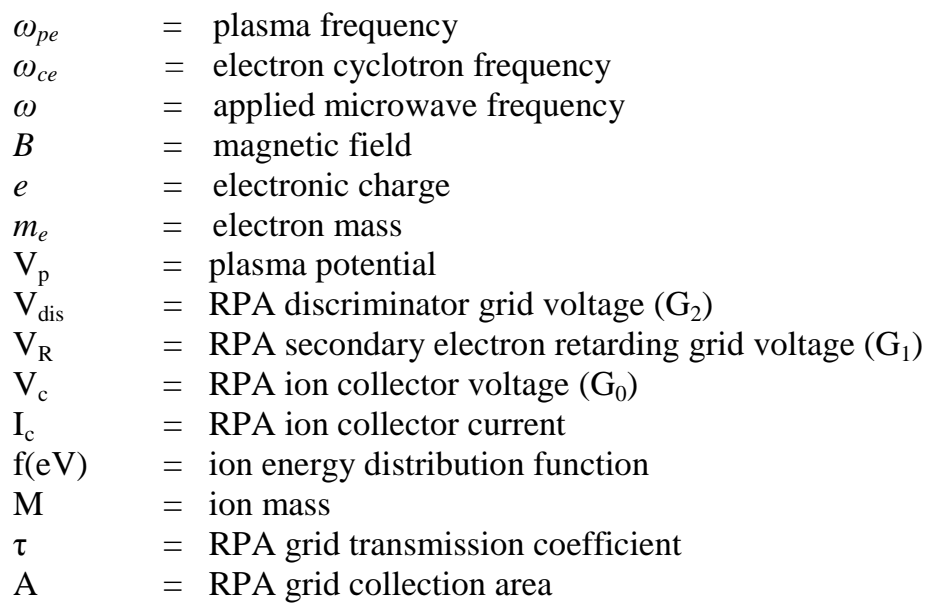

\section{Introduction}

$\mathrm{M}$

icrowave plasma sources driven by electron cyclotron resonance (ECR) have become established as viable plasma sources for applications in electric propulsion, primarily in the development of gridded ion thrusters ${ }^{1,2}$. ECR plasmas have many distinct advantages over traditional DC, hollow cathode-based sources ${ }^{3,4}$. These include electrodeless plasma production and instant on operation. Unlike hollow cathode discharges, ECR plasmas can operate with a variety of different propellants, including certain chemically reactive species. Furthermore, environmental protocols associated with hollow cathodes, which typically increase cost and handling complexity, are completely eliminated.

Furthermore, a gridless microwave thruster would have a number of desirable attributes that could circumvent some of the inherent shortcomings in a conventional ion thruster. Issues such as grid erosion, low thrust density, and complex optics system ${ }^{5}$ are all inevitable consequences of placing a high voltage acceleration grid within the thruster. In this paper, we present a microwave source designed to produce an ion beam without the use of a physical acceleration grid, hence bypassing the grid erosion and limited thrust density associated with conventional ion thrusters. The source, driven by electron cyclotron resonance absorption (ECR) in a magnetic field cusp geometry, produces plasma an expanding magnetic field with a strong gradient. Electrons from the neutralizing cathode travel

\footnotetext{
${ }^{1}$ Graduate Student, Department of Nuclear Engineering, 2355 Bonisteel Blvd., Ann Arbor, MI, 48109.

${ }^{2}$ Professor, Department of Nuclear Engineering, 2355 Bonisteel Blvd., Ann Arbor, MI, 48109.
} 
downstream toward the anode and are impeded by the strong field gradient. The magnetic field serves two purposes: first, to provide the resonant condition for ECR, and second, to confine / impede cathode electrons in such a way as to establish the ion acceleration zone. This latter mechanism is similar to the familiar magnetic layer acceleration zone implemented in Hall effect thrusters.

Ion energy measurements have previously been performed on a version of the device similar to the one presented here. In that experiment, the ion beam was unneutralized and relied on magnetic mirror forces to accelerate the ECR plasma out of the magnetic cusp. Despite the absence of a cathode, the results still indicated the formation of controllable potential differences in the ECR cusp plasma ${ }^{6}$ along with resulting ion beam emission. As can been seen from prior measurements, shown in Fig. 1, the peak ion energy closely matches discharge voltages $(40 \mathrm{~V}$, $60 \mathrm{~V}$, and $80 \mathrm{~V}$ ). In this paper, we present an improved design of the magnetic cusp acceleration concept, which includes a better isolated microwave feed and anode, and most importantly, the addition of a cathode electron source to provide neutralization of the ion beam.

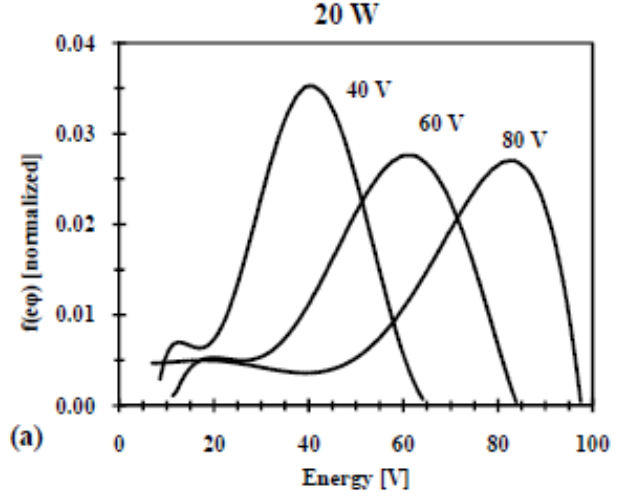

Figure 1. Previous ion energy measurements ${ }^{6}$ of an unneutralized ion beam created by the microwave source.

\section{Source Design and Experimental Setup}

\section{A. Theory of Microwave Device Design}

The primary design goal of the microwave device was to apply ECR to create a complete discharge circuit, with an ion beam produced by ECR and neutralized by a cathode electron source. In an ECR discharge, plasma is created in the region of magnetic field in which the electron cyclotron frequency matches the applied microwave frequency,

$$
\omega=\omega_{c e}=\frac{e B}{m_{e}}
$$

For the $2.45 \mathrm{GHz}$ source used in this experiment, the resonant magnetic field is $875 \mathrm{G}$. The practical implementation of this resonance condition requires a careful consideration of geometry and magnetic field design. If microwave power is launched from an external source, it will invariably approach resonance from the low field side. As a result,

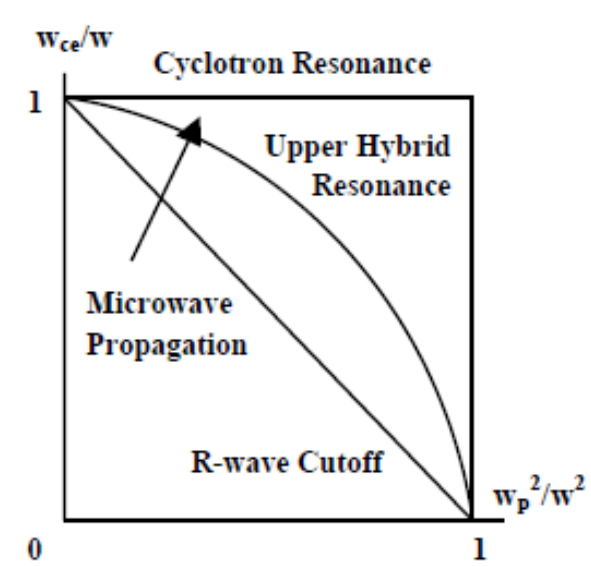

Figure 2. CMA diagram showing R-wave resonance and cutoff. Microwaves traveling from the low to high magnetic field will hit the cutoff before resonance.

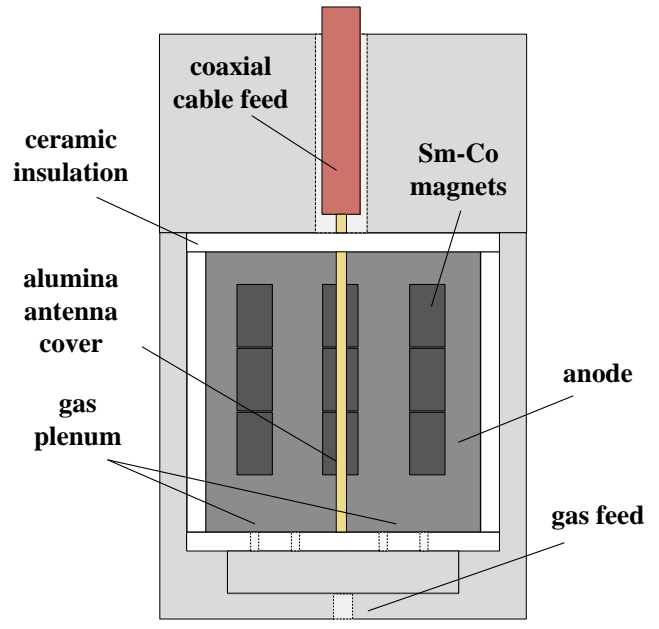

Figure 3. Microwave Device (Top View). Device shown without slot extraction cover. Ceramic walls and floor isolate the anode from the outer device body. 
microwaves will encounter the $\mathrm{R}$ wave cutoff before resonance and be reflected if it were not for tunneling into the upper hybrid resonance. Even in the case of tunneling, absorption at the upper hybrid resonance can only occur if the local plasma frequency is less than the microwave frequency. Otherwise, the microwave power will simply be reflected. These reflection and resonance processes are illustrated in the Clemmow-Mullaly-Allis (CMA) diagram shown in Fig. 2. The arrow represents the path taken by an externally launched microwave approaching ECR from the low field side. Note that in Fig. 2, increasing plasma frequency, wpe, and increasing electron cyclotron frequency, Wce, represent increasing plasma density and magnetic field respectively.

Efficient ECR production can be achieved, however, if an exciting antenna is placed at the root of the cusp. At this location, the field strength is larger than that required for resonance, allowing high to low field microwave propagation. This approach is employed in socalled distributed ECR plasma sources ${ }^{7}$, which have demonstrated over-dense plasma production deep inside magnetic cusps. Here overdense denotes the condition where the local plasma frequency exceeds the microwave frequency.

\section{B. The Compact Microwave Source}

The compact ECR device is shown in an overhead view in Fig. 3 and an inside cross sectional view in Fig. 4. Microwave power is provided by a copper antenna, covered by an alumina tube. The magnetic field is created using permanent samarium-cobalt (Sm-Co) magnets such that resonance occurs directly above (i.e. downstream of) the antenna. The magnets are aligned in three columns with alternating polarities, giving rise to strong confining fields between the columns and an expanding partially parallel field directly above the magnet within the cusp plasma.

The anode is isolated from the rest of the device (to the sides and from below) by thin ceramic macor plates. The ceramic plates and anode are housed in an aluminum outer device body, which is allowed to float electrically. This design allows the microwave antenna to be isolated from the ECR plasma and prevents charged particles from entering the anode without first traveling through strong magnetic field gradients. For this effort, the top of the device was covered with a thin stainless steel plate. An extraction slot, measuring $1 \mathrm{~cm} \times 3 \mathrm{~cm}$, was cut in the plate directly over the center magnet column (and antenna) to extract plasma current directly from the ECR plasma, as seen in Fig. 4. Argon gas was fed into the main device body from an isolated feed system that provided up to $32 \mathrm{sccm}$. Once inside the main device body, the gas was fed into a four hole gas plenum on the side of one of the ceramic walls in order to evenly distribute the plasma across each of the three magnets. The outside chamber pressure was measured using a thermocouple gauge for chamber pressures down to $1 \mathrm{mTorr}$ and an ion gauge for pressures down to $10^{-5}$ Torr.

\section{Discharge Circuit}

The compact ECR source was neutralized using a dual filament cathode constructed from $0.3 \mathrm{~mm}$ diameter tungsten wire. The filament current for the cathode was operated in the range 10-15 A. During basic operation, the discharge voltage was applied between anode and the cathode. The cathode served to neutralize the emerging ion beam and impede electrons motion as they approached the anode, hence leading to the establishment of the acceleration potential. The cathode electrons may also provide another source of ionization as they collide with neutral argon atoms on their way to the anode. The entire system was allowed to float electrically. 


\section{Ion Energy Distribution}

The energy distribution of the ions emerging from the ECR plasma was measured using a gridded retarding potential analyzer (RPA). A detailed description of probe theory and operation can be found elsewhere ${ }^{8}$. Figure 5 shows the commonly used three grid setup used in this experiment. This includes three gridded plates and a Faraday cup collector. Ion current from the source passes through the grids to the collector and is attenuated by biasing the middle grid (the discriminator, $\mathrm{G}_{2}$ ) at positive voltage $\mathrm{V}_{\mathrm{dis}}$, allowing only ions with energy greater than $\mathrm{eV}_{\mathrm{dis}}$ to pass to the collector. The front grid $\left(\mathrm{G}_{3}\right)$ is held at facility ground while the back grid (G1) is biased $\left(\mathrm{V}_{\mathrm{R}}\right.$ $=-30 \mathrm{~V}$ ) to prevent the escape of secondary electrons created by ion bombardment on the collector surface. Without the third grid, these electrons could escape the probe and be counted as erroneous ion current to the collector. The collector was biased negative $\left(\mathrm{V}_{\mathrm{c}}=\right.$ $-20 \mathrm{~V}$ ) to collect all ions passing through the discriminator grid $\mathrm{G}_{2}$.

The RPA produces an IV ( $\mathrm{I}_{\mathrm{c}}$ vs. $\left.\mathrm{V}_{\text {dis }}\right)$ curve that can be differentiated to yield the energy distribution $\mathrm{f}(\mathrm{eV})$ of the incident beam in terms of the ion mass $\mathrm{M}$, the electronic charge e, the transmission coefficient of the probe grids $\tau$, and the area of the probe collector A.

$$
f\left(e V_{d i s}\right)=\frac{M}{A \tau e^{2}} \frac{d I_{c}}{d V_{d i s}}
$$

In this work, ion energy measurements were taken over a range of different cathode current settings and pressure. Measurements were typically limited to the most operationally stable range of discharge voltages $(40-80 \mathrm{~V})$. Below discharge voltage of $40 \mathrm{~V}$, the collector signal was considered too weak while above $80 \mathrm{~V}$, the parasitic current due to presumably secondary electron emission prevented clean traces to be taken.

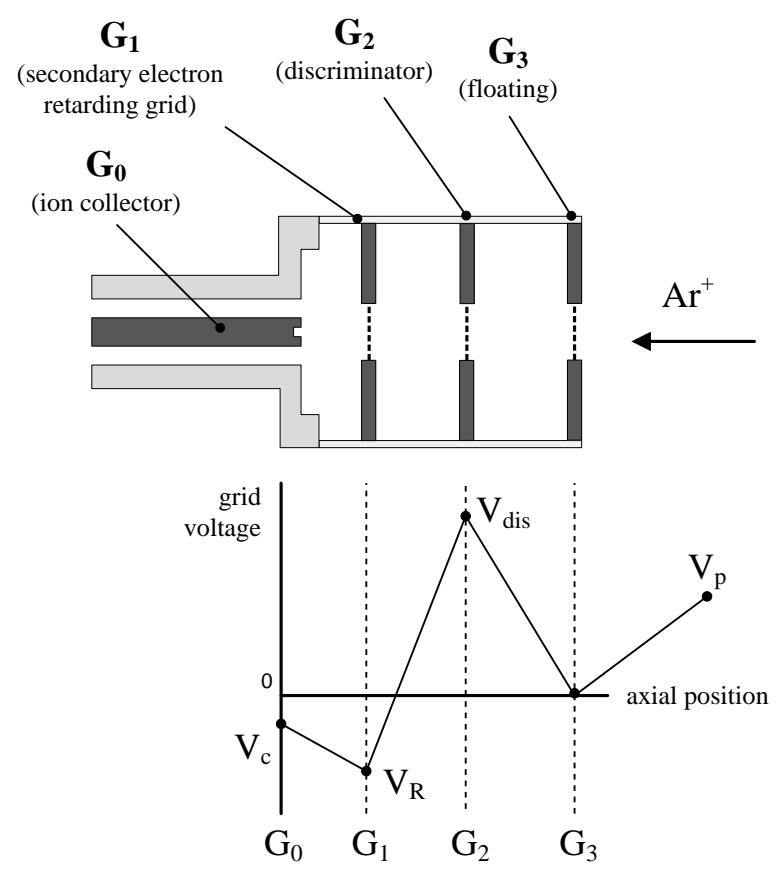

Figure 5. RPA diagram. Grids are from front to back: $G_{3}$, the floating grid, $G_{2}$, the discriminator grid, $G_{1}$, the secondary electron retarding grid, and $G_{0}$, the ion collector.

\section{E. Plasma Potential}

RPA curves can be used to determine the energy of the ions entering the RPA probe, but this may be quite different from the energy of the ions emerging from the source itself. As the ions exit the plasma and enter the front grid, they experience an additional energy gain while traveling from plasma potential to ground (the front grid $\mathrm{G}_{3}$ ). This additional energy must be subtracted from the RPA curves to determine the real ion beam energy emitted by the device. For this reason, the electrostatic potential of the surrounding plasma outside of the RPA probe was measured using emissive probes. The emissive probe is a well established electrostatic probe technique for measuring plasma potential, particularly in Hall thruster channels ${ }^{9}$ and magnetically confined plasmas ${ }^{10}$. The emissive probe is a practical alternative to plasma potential measurements extracted from a Langmuir probe IV characteristic, which typically don't saturate clearly in the presence on an applied magnetic field. The emissive probe is driven by a filament heater current. As this current is increased, the probe emits an increasing flux of electrons into the plasma until it eventually saturates and reaches the plasma potential. An emissive probe was constructed using $3 \mathrm{~mm}$ diameter double bore alumina tubing with $0.06 \mathrm{~mm}$ diameter tungsten wire for the filament, which had a loop radius of $1.5 \mathrm{~mm}$. The wire loop was connected to copper leads inside the alumina tube. The probe was powered using a $3 \mathrm{~A}, 20 \mathrm{~V}$ D.C. current controlled power supply.

\section{F. Ion Current Density}

A Langmuir probe was implemented to measure the ion current density directly outside the extraction slot of the device. This allowed an estimation of the actual ion beam current emerging from the ECR plasma. The Langmuir probe was constructed using $0.5 \mathrm{~mm}$ tungsten wire, partially covered by alumina tubing. The exposed area of the 
probe was $2.6 \mathrm{~mm}^{2}$. The Langmuir probe was placed $2 \mathrm{~cm}$ from the extraction slot directly over the center of the microwave device's extraction slot. The magnetic field at this location was 5 G. Ion saturation current was obtained by biasing the probe at $-40 \mathrm{~V}$.

\section{Results}

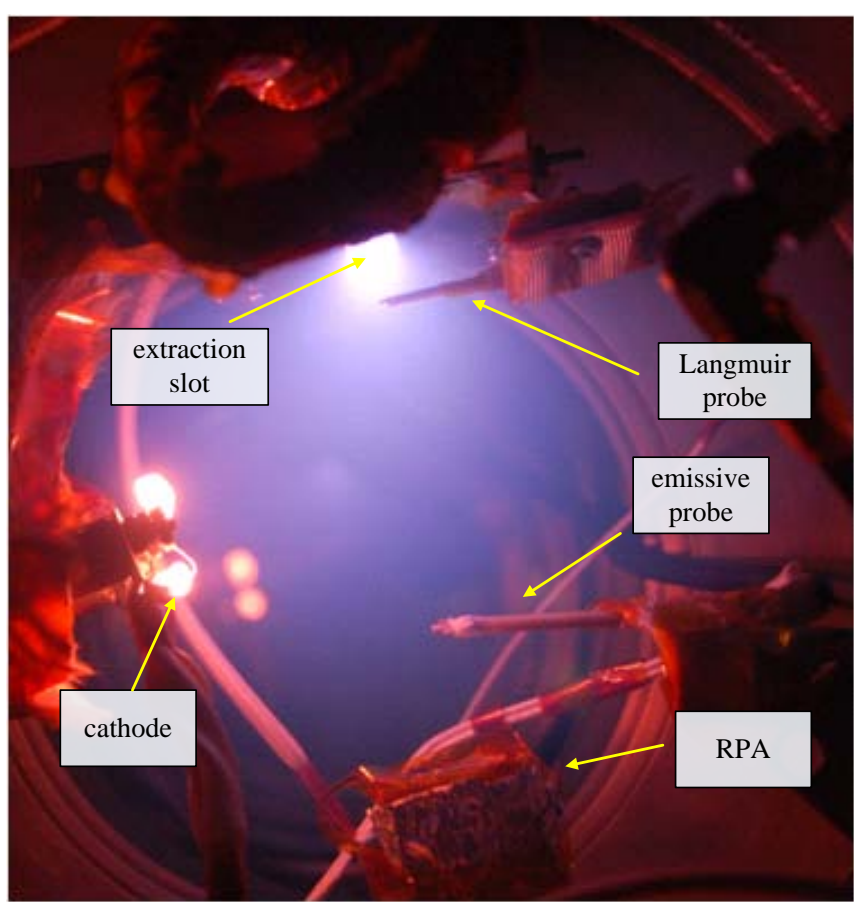

Figure 6. Typical operation of the microwave device. The plasma is shown emerging from the extraction slot. The probes are aligned to be on the centerline of the extraction slot.

\section{A. Basic Operation}

Figure 6 gives an overhead view of the microwave device, filament cathode, and probes during normal operation. The microwave device was observed to be self-starting at $35 \mathrm{mTorr}$. It was operated with the cathode in the $4-10 \mathrm{sccm}$ (1.8-5 mTorr) range. Cathode filament heater current was varied between 10-15 A. Device operation required a minimum absorbed microwave power of $50 \mathrm{~W}$. As the pressure was decreased, the device also required a minimum discharge voltage to sustain itself, typically around $20-40 \mathrm{~V}$. As the discharge voltage was increased above this value, the visible plume outside the extraction slot expanded reaching a uniform background glow similar to that seen in Fig. 6.

The most stable operating condition was determined to be $4.5 \mathrm{sccm}, 3.5$ mTorr. Under this condition, discharge IV traces were taken of the cathode operating under different emission currents. These are shown in Fig. 7. The discharge current was observed to increase monotonically with discharge voltage, indicating likely increased impact ionization as well as field driven collection of electrons at the cusp. The device was not operated above $80 \mathrm{~V}$ to

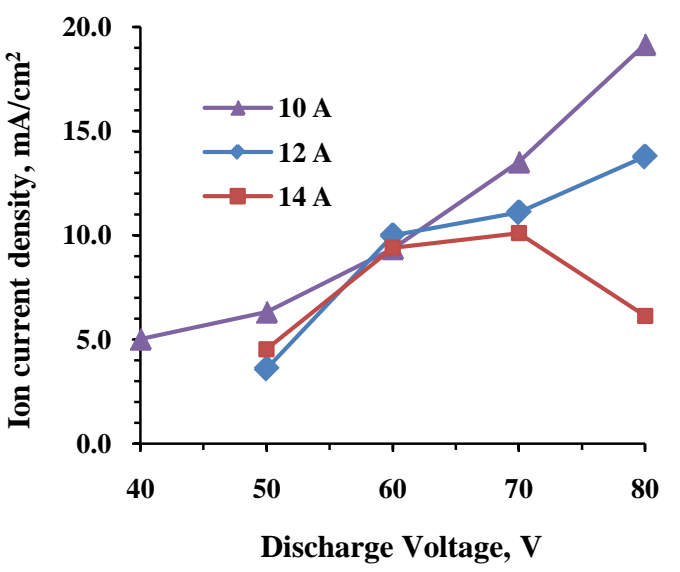

Figure 8. Ion current density measurements. The measured ion current density reaches up to $19.2 \mathrm{~mA} / \mathrm{cm}^{2}$. Current drops observed at the two highest levels may be due to antenna heating.
Figure 7. Discharge Current measurements. The measured discharge current is relatively constant across different levels of cathode driving current. For the highest level of filament current, the discharge current actually begins to decrease.

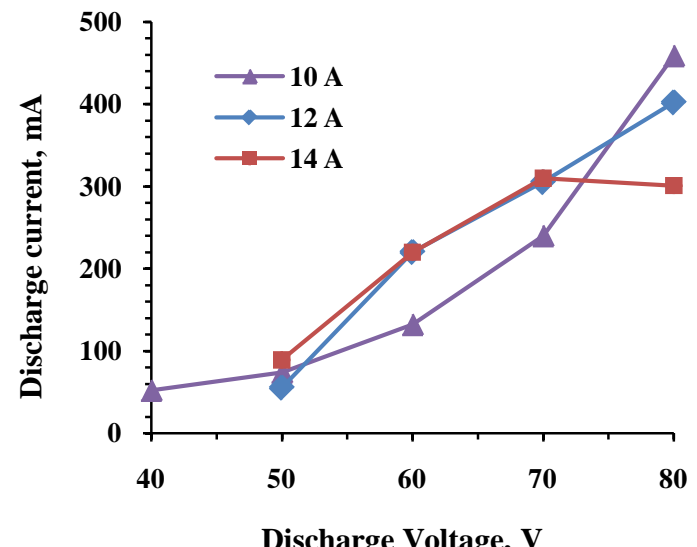

Discharge Voltage, $\mathrm{V}$ 
avoid overheating of the antenna.

\section{B. Ion Current Density}

Langmuir probe measurements of ion saturation current were taken to examine the flux of ions emerging from the extraction slot. These measurements were taken in the normal operating range, 10-14 A filament heater current, $3.5 \mathrm{mTorr}$ and are shown in Fig. 8. In the 40-70 V range of discharge voltages, the ion current behaves as expected, increasing with increasing discharge voltage. For the $10 \mathrm{~A}$ filament current, ion current density increases monotonically with discharge voltage, reaching up to 19.2 $\mathrm{mA} / \mathrm{cm}^{2}$. For comparison, the peak ion current density of a gridded ion thruster ${ }^{11}$ is below $5 \mathrm{~mA} / \mathrm{cm}^{2}$.

For the cases of higher filament heater current, the collected probe current actually decreases at high voltage. This effect is also observed in the measurements of discharge current (Fig. 7). The effect can possibly be explained by antenna heating. It may also be caused by a redistribution in the discharge voltage. If at this condition, a higher fraction of the discharge voltage appears at the cathode fall, then a smaller fraction will be available to extract ions from the device. To resolve this issue, spatial measurements of the plasma potential are needed.

The data from Fig. 7-8 can be used to estimate the ratio of ion beam current to discharge current, a figure of merit regarding device performance. Assuming that the ion current density is relatively uniform over the

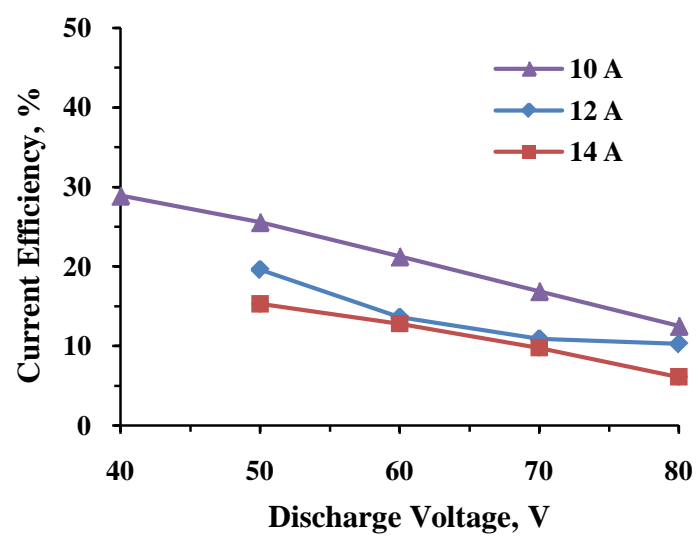

Figure 9. Calculated current efficiency. The efficiency decreases nearly linearly with increasing discharge voltage. extraction slot, we can estimate the total ion current emerging from the slot by multiplying the ion current density data in Fig. 8 by the slot area, which is $3 \mathrm{~cm}^{2}$. The current efficiency of the device can then be calculated as the estimate of calculated ion current, using Fig. 8, divided by the discharge current, from Fig. 7. Figure 9 shows the calculated current efficiency at the three levels of cathode driving current. The efficiency is observed to decrease linearly with increasing discharge voltage over all three levels of cathode driving current.

\section{Ion Energy}

Ion energy measurements were performed as a function of discharge voltage over the cathode filament current range 10-15 A, at 3.5 mTorr. The RPA traces, shown in Fig. 11, are normalized by their baseline current to more easily compare traces taken at different discharge voltages. At every level of cathode filament current, the traces indicate the presence of ion beams passing through the RPA collector. The knee of the RPA trace indicates the energy at which a significant fraction of ions are repelled from the collector. This can be taken as a measure of the mean ion energy entering the RPA. Plasma potential measurements, displayed in Fig. 10, taken using an emissive probe indicate that outside of the RPA, the potential remains constant (8.5 $\mathrm{V}$ for $10 \mathrm{~A}$ filament current and $17 \mathrm{~V}$ for $12 \mathrm{~A}$ filament current). For both cases, these values are well below the discharge voltage. From these observations, we can determine the characteristic energy of ions emerging from the extraction slot for the two specific cases of filament current, $10 \mathrm{~A}$ and $12 \mathrm{~A}$. In the case of $10 \mathrm{~A}$ filament current, we observe mean ion energies up to $54 \mathrm{eV}$ or about $77 \%$ of the discharge voltage. In the case of $12 \mathrm{~A}$, filament current, we observe mean ion energies up to $41 \mathrm{eV}$, or about $68 \%$ of the discharge voltage. The two remaining cases of filament current (12 A and $14 \mathrm{~A}$ ) indicate similar results.

A recurring observation among the RPA traces is the

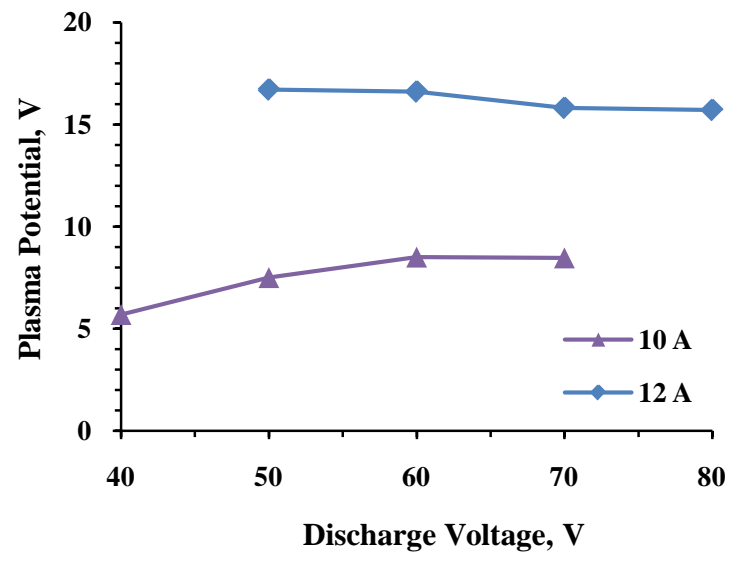

Figure 10. Plasma potential measurements. Measurements were taken directly outside of the RPA over the full range of operating discharge voltages $(40-80 \mathrm{~V})$. 


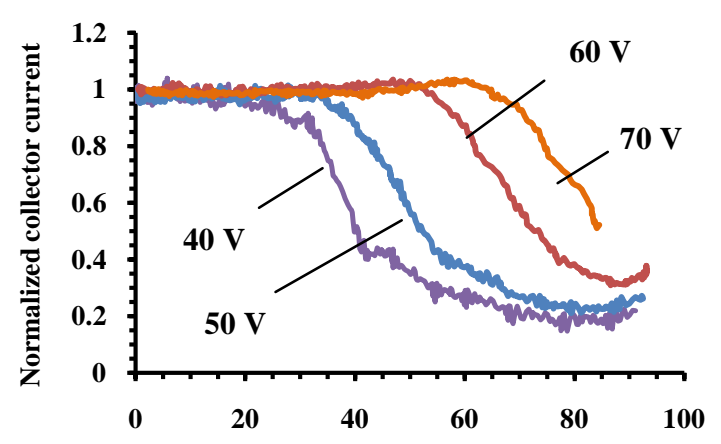

a.)

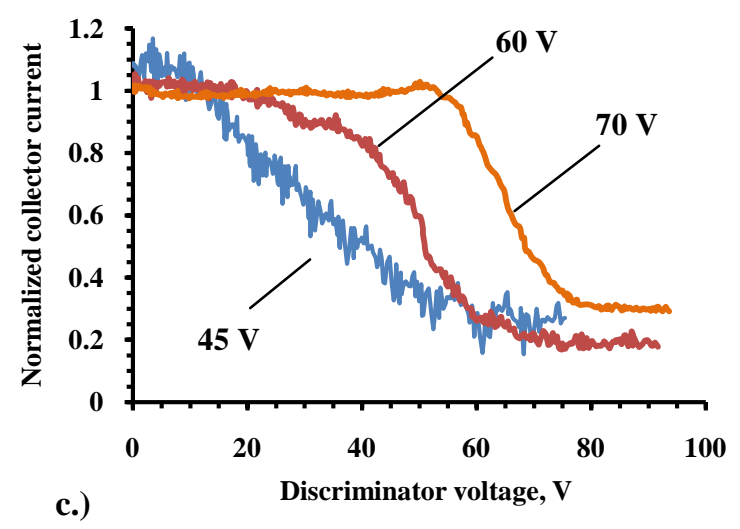

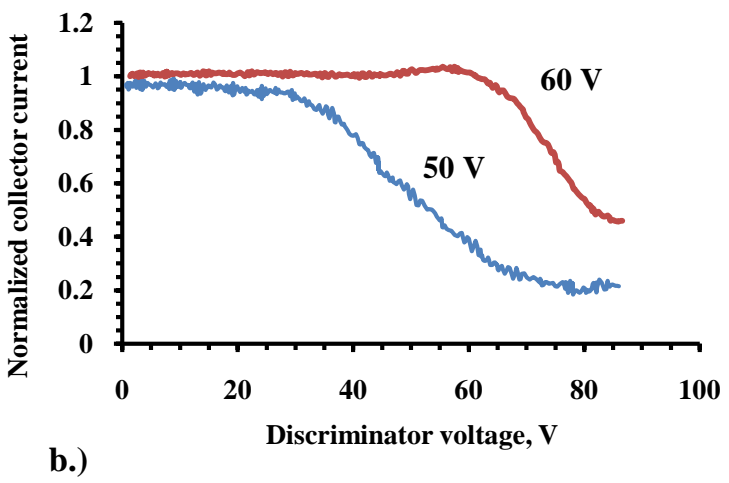

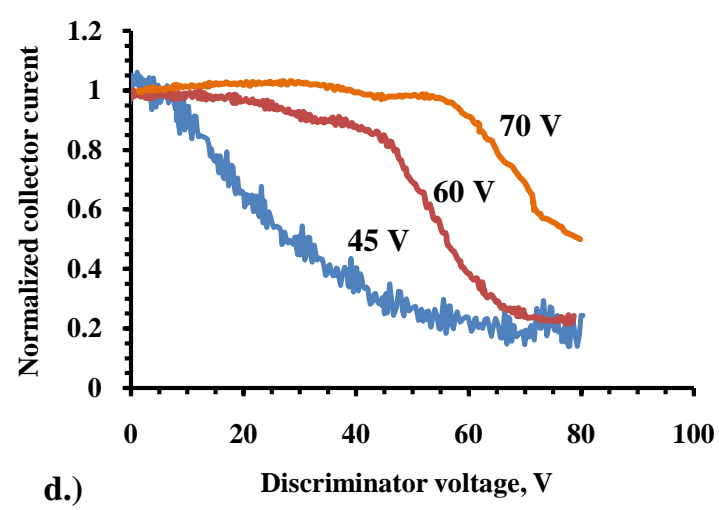

Figure 11. RPA measurements. Traces taken over four cathode filament heater current settings $a$.) $10 \mathrm{~A}, b$.) $12 \mathrm{~A}, \mathrm{c}$.) $13 \mathrm{~A}$, and d.) $14 \mathrm{~A}$. Voltage labels refer to the discharge voltage setting at each operating condition. Traces seen here are uncorrected for plasma potential.

energy distribution appears broad and dispersed. But as the discharge voltage is increased, distinct beam energies begin to appear at energies near the discharge voltage. This observation is consistent with the fact that the device is often observed to be unstable at discharge voltages $40 \mathrm{~V}$ and below, but becomes more stable with increasing voltage. The presence of ions with energies that are significant fractions of the discharge voltage, suggests that acceleration is occurring somewhere between the anode surface and the exterior region of the device.

\section{Conclusion}

In this effort, we have demonstrated the ability to create controllable ion beam energies in an ECR cusp plasma without the use of a physical acceleration grid. Energy measurements taken with an RPA indicate the presence of ion beams, with significant fractions of the discharge voltages, entering the RPA. This observation, combined with the measurement of relatively low plasma potential outside the device, suggest that ions are being accelerated to considerable energies inside the device or at least outside near the extraction slot. Moreover, ion saturation measurements with a Langmuir probe suggest that this beam density is as high as $20 \mathrm{~mA} / \mathrm{cm}^{2}$ outside of the extraction slot. These two observations suggest that the compact gridless ECR source could be used as a means to produce and accelerate ions for thruster applications.

It is still unclear over what region of the device the accelerating potential appears. Detailed measurements of plasma potential will need to be performed directly within the cusp at varying distances above the magnet and correlated with observed beam energy. This will provide insight into how the ECR plasma interacts with the cathode electron flux to produce a steady state accelerating potential. No attempt has yet been made to determine the effect of the field geometry and magnet orientation on the creation of ion beams in the cusp. In the future, the device will be further characterized by altering the shape and magnitude of the magnetic field in the expanding cusp. By optimizing the way the B-field restricts electron flow from the cathode to the anode, it should be possible to significantly improve the current efficiency from levels observed in this experiment. Given that the ion acceleration zone is largely determined by the magnetic field geometry, which impedes cathode electron flow, this is expected to be a major influence on the device's performance and efficiency. 


\section{References}

${ }^{1}$ Kuninaka, H., Funaki, I, Nishiyama, K., "Result of 18,000-Hour Endurance Test on Microwave Discharge Ion Thruster Engineering Model”, Proceedings of the $36^{\text {th }}$ Joint Propulsion Conference \& Exhibit, 2000, AIAA-2000-3276.

${ }^{2}$ Foster, J.E., Haag, T., Patterson, M. “The High Power Electric Propulsion (HiPEP) Ion Thruster”, NASA/TM, 2004213194

${ }^{3}$ Kim, S., Okamoto, H., Sugiki, T.M., “Endurance Test of Microwave Engine”, Journal of Propulsion \& Power, Vol. 22, No. 5, 2006.

${ }^{4}$ Takao, Y., Miyamoto, T., Yamawaki, K., “Development of ECR Microwave Discharge Ion Thruster,” Vacuum, Vol. 65, No. 21 May. 1984, pp. 361-366.

${ }^{5}$ Van Noord, J.L. "Lifetime Assessment of the NEXT Ion Thruster", Proceedings of the 43rd Joint Propulsion Conference \& Exhibit, 2007, AIAA 2007-5274.

${ }^{6}$ Sommers, B.S., Foster, J.E., "Characterization of Acceleration Processes in a Surface ECR Plasma Source”, Proceedings of the $44^{\text {th }}$ Joint Propulsion Conference \& Exhibit, 2008, AIAA 2008-4536.

${ }^{7}$ Pichot, M., Durandet, A., "Microwave Multipolar Plasmas Excited by Distributed Electron Cyclotron Resonance: Concept and Performance”, Rev. Sci. Instruments, Vol. 59, No. 7, July 1984, pp. 1072-1075.

${ }^{8}$ Kudyan, H.M., "Interpretation of electrostatic energy analyzer data of flowing plasma”, Rev. Sci. Instrum., 49(1), Jan. 1978.

${ }^{9}$ Haas, J.M., Gallimore, A.D., "Internal plasma potential profiles in a laboratory-model Hall thruster”, Phys. of Plasmas, Vol. 8, No. 2, Feb. 2001.

${ }^{10}$ Hershkowitz, N., Cho, M.H., "Measurement of plasma potential using collecting and emitting probes”, J. Vac. Sci. Technol., A 6 (3), May 1988.

${ }^{11}$ Frandina, M., Arrington, L., "Status of the NEXT Ion Thruster", Proceedings of the $41^{\text {st }}$ Joint Propulsion Conference \& Exhibit, 2005. AIAA-2005-4065. 